\title{
Contents
} Frontiers in Deep-Earth Physics
by J.-P. Poirier

POROUS SILICON LUMINESCENCE: Voltage Control of the Colour

\section{FUSION THEORY INSTITUTE:}

Theory Development Needed

The European Spallation Source

Electronic Publishing Expands

CONFERENCES \& SCHOOLS

STUDENT MOBILITY:

The Soros Foundations Cooperate with EPS

NEWS FROM BRUSSELS:

Library aid, ERCs, ERASMUS, Large facilities, East-West deadlines

- Europhysics Notes ICTP Director, ELFE

\section{European Physical Society}

Europhysics News is the journal of the European Physical Society that acts through Divisions, Sections and Groups to promote collaboration among physicists. Subscription price: SFR 135.- per annum.

\section{Editorial Board}

P. Bochsler, Bern University

S. Ciliberto, ENS, Lyon

A.-J. Dianoux, ILL, Grenoble

Editor: P.G. Boswell

Exec. Sec.: G. Thomas

T. Jarlborg, Geneva University

J. Schacher, Bern University

M. Siegrist, EPFL, Lausanne

President: N. Kroó KFKI, Budapest

\section{Editorial and Advertising}

EPS Secretariat, P.O.Box 69

CH-1213 Petit-Lancy 2, Geneva

Telephone: $\quad+41(22) 7931130$

Telefax: $\quad$ +41 (22) 7931317

E-mail: epnews@cernvm.cern.ch

Advertising is charged according to space, on a sliding scale ranging from SFR 2700.- for a full page to SFR 700.- for a one-sixth page. Rates for four-colour and cover advertising, and series insertions, on application. Special rates for Associate Members of EPS; $25 \%$ reduction for announcements of situations vacant.
Deadline for advertising orders:

first week of month of publication.

Subscriptions \& EPS Fees:

Société de Banque Suisse,

Geneva; Acc. No. 164.899

Swiss Post Office Acc.: CCP

Geneva 12-19107-4

German Post Office Acc.: Postbank

Karlsruhe 1801-30-754

Eurocheque to EPS, Geneva

EPS Budapest Secretariat

Nádor u. 7, H-1051 Budapest

Telephone: $\quad+36$ (1) 1173510 Telefax: $\quad+36(1) 1176817$

\begin{abstract}
Cover illustration
The Earth's layers: the upper mantle is mostly composed of silicates of magnesium and iron (olivine and pyroxenes). The lower mantle has the same chemical composition as the upper mantle but is composed of a high-pressure silicate phase of perovskite structure and of oxides of magnesium and iron. The outer core is liquid iron with some lighter elements. The inner core is solid, almost pure iron. The layers are separated by discontinuities in density and seismic velocities (J.-P. Poirier, p. 25).
\end{abstract}

[From J.-P. Poirier, Les Profondeurs de la Terre (Masson, Paris)].

D.F. Düchs, formerly head of the Theory Division at JET and now with the Max Planck Institute for Plasma Physics, Garching, argues that Europe requires institutionalized collaboration at the EURATOM Association level to reverse the trend in fusion plasma research away from theoretical analysis towards numerical application of theory.

\section{Theory Development Needed}

"You must admit that fusion plasma theory has so far been rather unsuccessful". Such remarks have regularly been put to me by colleagues from fusion plasma engineering and experimental physics. The immediate reaction is to point out fusion theory's successes, even in the very applied environment of institutions such as the Joint European Torus (JET) where most theoretical and computational skills have to support the evaluation of data. Here are a few examples:

- Practically all tokamaks have been designed, within given financial and technical limits, on the basis of theoretical models and predictive computations using computer codes for heating, losses and plasma stability. Notwithstanding some deficiencies, these codes have had a very significant impact on decisions and can claim a large share of the success achieved with these devices.

- The analysis of ion-cyclotron heating started at JET with rather primitive (and incorrect) models which evolved into sophisticated codes that treat the complex interaction between electromagnetic wave propagation and the responding plasma particles.

- Neoclassical transport theory has been refined several times to yield, for instance, an order of magnitude increase in electron heat conduction and suitable theories for impurity and improved transport.
- Recently, we succeeded in computing three-dimensional magnetic fields with the accuracy necessary to assess the impact, on the plasma, of errors in the applied fields.

However, it cannot be denied that many essential features of fusion plasmas are not yet understood, either qualitatively with respect to the underlying physical mechanisms, or quantitatively in seeking agreement with measured data (for those cases where mechanisms can be formulated in equations but which are not too difficult to solve). Wellknown examples of situations for which a proper theory is lacking include "anomalous" transport, where fluxes of particles, momentum, and heat across the magnetic field are much larger than predicted, "sawtooth oscillations" where the peak plasma temperature suddenly collapses, and "density limits" where the plasma is extinguished when one tries to feed in more particles.

Comparisons with the state-of-the-art in the 1950's and 1960's show that plasma theory has undoubtedly made enormous strides without which controlled thermonuclear fusion research would not be as far advanced as it is today. Nevertheless, the spectacular improvements in parameters of merit, plasma temperature $T$, and confinement product $n \tau$ for a reaction time density $n$ and an energy confinement time $\tau$ are pre- dominantly due to empirical methods and engineering intuition. Although sometimes wasteful, these methods have fortunately been largely successful up to now despite the lack of a proper theoretical understanding of the details of the physics of plasmas. The situation raises several important questions: - Is theory necessary for fusion research after the empirical approach has brought such obvious progress?

- Why is there still no theory for important phenomena even after 40 years of research? - If theory needs strengthening, what measures would improve its development?

\section{Theory is Necessary}

The question concerning the need for adequate theory is obviously rhetorical. Scientists and engineers will pass away, experimental devices decay; only true theory has a chance of living on. Even an empirical tinkerer follows a theoretical concept, although usually one that is far short of quantitative scientific rigour. Empirical rules and formulae often substitute for complicated theory. However, empirical formulae only summarize and interpolate existing data; any extrapolation based on empirical results alone bears an intrinsic risk of failure. Future fusion experiments will be very expensive so even minor errors will be costly; any progress 
in theoretical understanding reduces the risks accompanying extrapolation, and can thus play an economic rôle. Take, for example, the dependence of the cost $\left(\approx 5 \times 10^{9} \mathrm{ECU}\right)$ of a future fusion device, say the International Fusion Test Reactor ITER, on plasma confinement and the ionic impurity level. A recent study [Fusion Engng \& Design 15 (1992) 291] concludes that the cost $C$ of ITER is approximately proportional to $Z_{\text {eff }} / h^{1.3}$ where $h$ measures the improvement over the usually observed energy confinement time $\tau_{\mathrm{EL}}\left(h=\tau_{\mathrm{E}} / \tau_{\mathrm{EL}}\right)$, where $\tau_{\mathrm{E}}$ is the actual future confinement time of ITER). The effective electrical charge of the ions, $Z_{\text {eff }}$, averages over all ions in the plasma, i.e., including non-hydrogenic impurities.

In order to maximize the chance of success, one is obliged to design the device for pessimistic values of $h$ and $Z_{\text {eff. }}$. Any change $\left(\Delta h, \Delta Z_{\text {eff }}\right)$ in these two parameters affects the cost according to

$$
\Delta C / C \approx-1.3 \Delta h / h+\Delta Z_{\text {eff }} / Z_{\text {eff }} .
$$

Improvements of only $10 \%$ in $h(\Delta h>0)$ and $Z_{\text {eff }}\left(\Delta Z_{\text {eff }}<0\right)$ give $\Delta C / C \approx 0.23$, i.e., a saving of almost one-quarter - a large amount in view of the enormous cost. Incidentally, should either $\Delta h$ or $\Delta Z_{\text {eff }}$ turn out to have the opposite sign, the whole device would very likely be a failure and therefore the saving accruing from a good prediction would be even larger.

Even 20 years of theoretical work costing $20 \times 3 \times 10^{6}=6 \times 10^{7} \mathrm{ECU}$ would be negligible in relation to the savings.

Pessimists might argue that for the problem of anomalous transport (i.e., where $\Delta h$ and $\Delta Z_{\text {eff }}$ are significant), the chance of finding a theoretical solution is as low as was the case for hydrodynamic turbulence. I believe, however, that some progress can probably be achieved already by using more mathematically advanced methods to evaluate existing data.

\section{Plasma Theory Remains Incomplete}

The question as to why plasma theory has remained incomplete is a much more serious issue. Progress in theory hinges in the first instance on theoreticians, and one is tempted to attribute failures to their ineptitude. Worldwide, well over 5000 scientists and engineers are working on controlled fusion, with up to about $10 \%$ being concerned with theoretical topics. So at least 20000 man-years of work have been spent on fusion theory, indicating that an enormous number of calculations have been made.

With respect to the quality of scientific craftsmanship, intelligence, creativity, ingenuity, diligence, etc., the average plasma theoretician is definitely not inferior to colleagues from fusion engineering, experimental physics, and other fields of physics; simple statistics would suggest that there must have been a few excellent minds at work.

If it is not the theoreticians who are at fault, then it can only be the unassailable nature of the research object. In analyzing fusion research we should heed three dominant and distinctive characteristics of plasma theory: - With negligibly few exceptions, it is exceedingly complex; many interacting effects must be taken into account simultaneously in practically all cases.

- Interaction is not only local but global, implying that particles and other components located far apart influence each other.

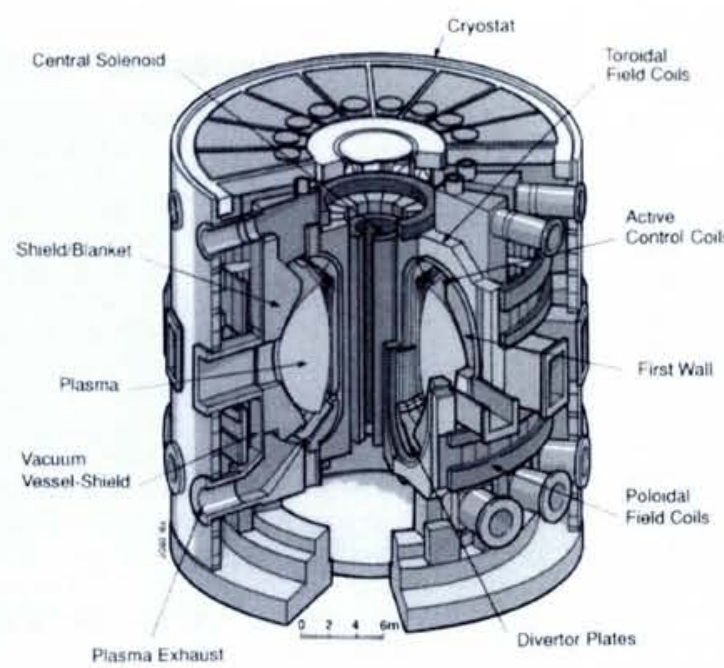

The conceptual design of the ITER tokamak reactor. The tokamak is the most successful confinement system for plasmas relevant to controlled thermonuclear fusion. Ideally, the magnetic field and plasma configuration is toroidal with axial symmetry. The toroidal (and largest) magnetic field component is produced by (applied) currents through a set of toroidal field coils. A toroidal plasma current is driven by transformer action with the transformer coils as primary and the plasma as secondary circuits. This current creates its own poloidal (perpendicular to the toroidal) magnetic field component. Additional currents in poloidal field coils interact with the plasma current to position the plasma and to shape its vertical cross-

section. By properly choosing the currents in the external coils the magnetic field arranges itself in nested toroidal surfaces with axial symmetry in which the plasma is caged.

- Most interactions are highly nonlinear so that continuous feedback must be treated.

An important consequence is that the solutions of theoretical problems cannot generally be split up into independently tractable subproblems which are then summed to provide a comprehensive solution.

Hydrodynamics, an area of classical physics also has some of these features and can indeed be considered as a sub-field of fusion plasma theory. There too, after more than a century of research, solutions for problems arising for example in turbulence and weather forecasting are still unavailable. Indeed, some of the most outstanding brains in physics have unsuccessfully tested their powers in the area before moving on to more accessible topics.

Recalling hydrodynamics also offers a glimmer of hope. After a long period of slow progress, large strides forward came with the advent of electronic computers. Although a few diehard traditionalists still dispute the rôle of numerical calculations in theory, it seems trivial to recognize the computer as an extremely powerful new tool. Systems of equations previously considered inconceivably large can now be treated, and extensive iteration procedures are possible. Both aspects are obviously essential for attacking complex, nonlinear problems.

Secondly, major advances in many research areas have often been associated with the discovery and development of new tools and with the refinement of diagnostics. In practice, many theoreticians learn one or more mathematical methods and then spend considerable time looking for suitable problems which can be solved using these methods. This approach, together with the large number of man-years spent, means it is likely that all learnable, i.e., all available traditional ("analytic") methods, have indeed been applied to all major questions in fusion theory. Hence, the traditional methods of theory do not seem to be shrewd enough for some major problems in fusion research; new mathematical methods must be introduced before advances can be expected.

\section{The Situation is Not Good}

It must be stressed that the development of theoretical tools has, of course, been going on in the past (the application of computers is abundantly obvious and my own contributions to fusion theory have been intimately connected with novel numerical methods). However, such activities have been at best tolerated by those directing the fusion effort and their development has definitely not been encouraged. This attitude was widespread and indeed intensified during the past decade. The narrow task given to JET's Theory Division was explicitly “... the application of current theory to JET problems" [J. Teillac, JET Council, 1979]; the same is true of the project for the Next European Torus NET that was to have followed JET. Theory divisions have shrunk and are now severely overage in many of EURATOM's national Associations. Largely at their expense, growth has been directed towards application-orientated groups closely linked with local experimental projects.

There is no doubt that expensive experiments need detailed theoretical support. Conversely, experimental observations provide an invaluable stimulus for new theory and give an immediate indication of the relative importance of possible phenomena. $\mathrm{Ne}$ vertheless, the enforced strong orientation towards application must lead to theory drying up and a serious consequence of the shift in emphasis is that plasma theory in the universities has been tagged as "applied" (i.e., relatively non-academic), and a number of plasma physics chairs have already been lost. The way in which theory is funded also works along the same lines: it is considered more advantageous to play safe and produce a report with applications to experimental results than to embark on some new theoretical model where the outcome is risky.

Applied numerical mathematics and computation afford much scope for improvement since a very large portion (well over half according to my observations) of theory-related computations must be considered dubious. An error estimate is hardly ever even attempted; simple numerical methods are usually applied on an intuitive basis; minimizing computer time is often considered to be more important than the mathematical requirements of stability and convergence. I have in fact seen extensive calculations where not even the basic conservation laws (conservation of mass, energy, etc.) were reproduced.

Overall, it seems that fusion theory has 
shifted too far towards application, forgetting the development of general theory, especially the relevant mathematics.

\section{There Are Ways Forward}

New methods can be made accessible to fusion theory either by transferring suitable methods from other areas or through origina development. Concerning the first, obvious areas are the neighbouring fields of plasma physics, e.g., low-temperature plasmas, inertial fusion, and extraterrestrial plasmas; then follow fluid theory and gas dynamics. In contrast to present-day practice, each should be regularly scrutinized by plasma physicists and co-operation actively pursued; their representatives should be invited to assess fusion theory problems.

With respect to the second approach, very few people develop or at least advise on the application of methods of a more mathematical nature. In contrast to the situation in the 1960 's, no fusion laboratory has been keen to finance appointments along such lines.

Without trying to devise a detailed research programme (which should not be too difficult) a few general examples will illustrate that there are suitable topics for investigation. Consider theoretical tools: there exists a huge backlog of barely investigated computational procedures which have been and are being used for analyzing fusion data. Mathematical scrutiny leading to error estimates for published computational results are a scientific necessity and would provide an extensive work programme. On the physics side, the main unsolved problems can be illustrated using instabilities as an example. A large variety of unstable plasma situations has been discovered by applying traditional (linearised) stability theory to the various types of models for plasma (kinetic, fluid, mixed, etc.). The criteria obtained for the possible onset of instability are, of course, very valuable - but they are only the first step. One ultimately needs to know about the advanced stages, i.e., the large-amplitude behaviour and the saturation levels of the instabilities, which are determined in most cases by nonlinearities and by the growing interaction with effects that may have been negligible in size during the initial phases. Such "advanced stages" have only been calculated for a few cases.

More generally, plasma theory research has been extended in the past into critical and difficult directions characterized by the the properties of plasmas (complexity, global behaviour, nonlinearity). Remarkable success has been achieved - albeit mostly computationally and only in isolated cases but it is the basis for optimism. Some of these cases are used below as examples and possible starting points for further development within the relevant group of problems.

Many problems involving three or more dimensions or lack of symmetries remain open. For example, tokamak plasma equilibria are still taken as axially symmetric although nonaxisymmetric structures (magneto-hydrodynamic modes, "snakes", periodic field ripples, etc.) have regularly been observed. MHD equilibria with flow are still, in general, unsolved; three-dimensionality introduced by external heating, pellets and recycling is obvious. Lack of symmetry can cause the destruction of surfaces to which plasma particles are bound i.e., it may be difficult to

\section{Institute for Fusion Studies}

The Institute for Fusion Studies (IFS) is a national centre for research in theoretical plasma physics and controlled thermonuclear fusion. Its mandate is to study theoretical physics issues arising in a variety of controlled fusion contexts, with special emphasis on fundamental problems of long-range significance. The IFS also serves as a centre for information exchange, nationally and internationally, by arranging visitor programmes, courses, conferences, and workshops. As part of this effort, it is the principal site in the USA for the exchange activities of the US-Japan Joint Institute for Fusion Theory. In addition, the IFS plays an important rôle in training students and postdocs.

The research being pursued at the IFS is diverse, including such topics as plasma turbulence, transport theory, numerical simulation, stability theory, and nonlinear plasma dynamics. Much of this work is interdisciplinary, involving neighbouring fields of research, such as mathematical physics, fluid dynamics, statistical mechanics, and space physics.

Currently, the IFS has a staff of some 25 Ph.D. scientists (including physics faculty, research scientists and postdoctoral fellows) and approximately 20 doctoral students, in addition to computer programmers and administrative personnel. It sponsors a vigorous visitor programme, with close to 10 visiting scientists typically in residence. The IFS actively supports numerous research collaborations with laboratories and universities in the US and other countries.

compute the distribution of particles. One easily imagines the difficulty to refine distribution functions in up to six-dimensional phase space if a plasma phenomenon needs to be treated by kinetic theory.

The basic problem of MHD equilibrium also serves as an example of nonlinearity. Nonlinear equations do not necessarily have a unique solution so one asks what solution will be produced by a numerical scheme, and what conditions will make it the physical one. We can think here in terms of bifurcation and multifurcation where it is possible to build upon existing results.

Another open area is nonlinear coupling at vastly different scales of space and time. Examples are "dissipative MHD", nonlinearly connected electrical or thermal conduction with wave propagation, asymmetric particle drifts, connecting gyroradii with the circumference of the plasma torus, and the interactions between ions and electrons connecting very different particle masses.

\section{A Possible Solution}

As indicated earlier on, the European groups developing fusion theory either consist of only a few people (e.g., those in universities) or have been thinned out so that even the largest is now below critical size (which admittedly depends on the individuals involved). Plasma theory, however, is so extensive that theorists must specialize and because of the non-additivity, intensive contacts with other specialists are necessary. It would do no harm to rebuild several theory groups but given the present circumstances it seems more appropriate to look for a "minimum solution" which at least ensures survival. Experience shows that collaboration over large distances is usually inefficient and expensive. In addition, it seems to work only between a few partners and is directed and streamlined with difficulty.

Strong coordination and concentration on the needs of fusion theory is necessary because any expansion of the research basis entails the risk of the whole effort becoming too diffuse. This points towards the creation of a centralized European institution as the most economical and efficient minimum solution. With the JET Project, the European Union (EURATOM) has proved that it can successfully run community-wide efforts. EURATOM would therefore appear as the logical framework for a centralized institution - for a European Fusion Theory Institute.
The proposed expansion could in principle be done within an Associated Laboratory. However, over and above the argument for centralization, the following points would not favour such a route:

- It is more difficult (and far less efficient) to change the established direction of an existing theory group than to start a new one.

- Each Association has in the past directed its theory programme primarily according to its own needs. More general European requirements, especially those of external and small groups, have been considered with, at best, low priority; there are few realistic possibilities for influencing programme decisions from outside.

- In comparison with engineering and experimental aspects, theory must catch up so the total theory effort (i.e., the number of posts) should increase. But the proposed institute should not be formed by simply regrouping as existing theory groups in the Associations must certainly not be reduced in size.

- In fusion theory, as in any research area, some problems are more amenable while others are more difficult to solve. Without centralization, given the relatively loose, sideby-side co-existence of the Associations, there would inevitably be a run on the easier topics with costly duplication of work. A theory institute set up within the EURATOM framework would on the other hand guarantee general access to, and overall influence on, the research programme and enhance the total theory effort in Europe.

The institute approach perhaps represents an ideal solution. Without compromising the basic ideas one can easily consider other forms of organization, and certainly one could start with a low-cost solution involving a small permanent staff. The College on Plasma Physics held from time to time at the International Centre for Theoretical Physics, Trieste, where fusion plasma theorists collaborate for several months may serve as an example (based with one of the Associations, most of the funds could probably be spent on travel expenses and computing).

I have restricted my analysis to the situation in western Europe but it is worth pointing out that the situation was not very different in the USA, where the importance of supporting fusion theory in general has been recognized, and the Institute for Fusion Studies (see insert) at The University of Texas founded. Europe could certainly learn from this experience. 